\title{
Comparação antropométrica e somatotípica de jovens tenistas a partir da lateralidade
}

http://dx.doi.org/10.11606/1807-5509202000010113

\author{
Geovane KRÜGER* \\ Elisa Cristina LEMOS* \\ Lucas Garcia FERREIRA* \\ Ricardo Dantas de LUCAS*
}

*Universidade Federal de Santa Catarina, Florianópolis, SC, Brasil.

\section{Resumo}

Este estudo teve como objetivo determinar e comparar o perfil antropométrico e somatotípico de jovens tenistas classificados em dois grupos maturacionais. Também foi objetivo comparar as variáveis antropométricas entre os lados dominante e não-dominante, e comparar o perfil somatotípico calculado a partir de medidas obtidas nos lados dominante e não-dominante. A amostra foi composta por 23 jogadores de tênis do sexo masculino com idades entre 11-17 anos. Os participantes foram classificados em dois grupos a partir do cálculo da distância do pico de velocidade de crescimento em estatura (PVE). Os jogadores que apresentaram valor do PVE inferior a 0 foram incluídos ao grupo denominado PRÉ ${ }_{\mathrm{PVE}}$ $(n=9)$, enquanto os sujeitos com valor maior ou igual à 0 foram classificados como PÓS $S_{\text {PVE }}(n=14)$. As variáveis antropométricas e os componentes do somatotipo foram calculadas a partir de medidas obtidas em ambos os lados do corpo. Foram observadas assimetrias (diferença significante entre os lados) de diâmetro ósseo de punho e dobra cutânea tricipital no grupo PÓS $\mathrm{PVE}$ assimetrias de diâmetro ósseo de úmero e perímetro de braço contraído em ambos os grupos. 0 somatotipo médio calculado para o lado dominante do grupo $\mathrm{PRE}_{\mathrm{PVE}}$ foi 2,9 - 4,5 - 3,7 e para o lado não-dominante 3,0 - 4,3 - 3,7, ambos classificados como ectomesomorfo. 0 grupo PÓS ${ }_{\text {PVE }}$ para o lado dominante foi classificado como ectomesomorfo $(2,8-4,4-3,5)$, e o lado não-dominante como mesomorfo-ectomorfo $(2,9-4,0-3,5)$. Em esportes com predominância unilateral deve-se ter cautela no cálculo do somatotipo a partir do lado direito, como é proposto originalmente, podendo levar a uma interpretação equivocada do perfil somatotípico do atleta, especialmente para aqueles com maturação somática avançada.

Palavras-Chave: Tênis; Antropometria; Somatotipo; Assimetria; Composição Corporal.

\section{Introdução}

O Tênis é um dos esportes mais praticados em todo o mundo, tanto no nível profissional quanto ao nível recreacional ${ }^{1,2}$. A prática deste esporte provém muitos benefícios, tais como, versatilidade de padrões de movimento, melhora nas funções cardiovascular, musculoesquelético e metabólicas ${ }^{1,3}$. No âmbito competitivo, os jogadores necessitam produzir golpes com maior potência e possuir um alto nível de aptidão física, de modo a conseguir manter a alta intensidade de jogo durante longos períodos ${ }^{4}$.

As medidas antropométricas fornecem informaçôes úteis sobre parâmetros da composição corporal e possuem considerável aplicação para compreender os requerimentos de um esporte. Possuem estreita correlação com características fenotípicas específicas dos atletas, que são obtidos em parte pela análise do seu perfil antropométrico, o qual serve para realizar comparações com outros atletas da mesma modalidade e/ou como controle longitudinal de seus próprios valores ao longo da temporada ou carreira ${ }^{5,6}$.

Fatores morfológicos possuem grande influência no desempenho esportivo, sendo que atletas adultos de elite têm características 
físicas e fisiológicas especificamente adequadas para o seu esporte ${ }^{7}$. Sendo o Tênis um esporte de predominância unilateral, alguns autores têm encontrado assimetrias de densidade mineral óssea e da massa muscular de braço ${ }^{8-11}$ em decorrência da demanda do esporte, sendo estas maiores no membro superior dominante em relação ao não-dominante ${ }^{10,12}$. Desta forma, comparaçóes lado-a-lado podem nos ajudar a compreender os mecanismos que afetam o aumento de assimetrias a longo prazo em esportes com predominância unilateral.

O estudo do somatotipo é utilizado para identificação de perfis morfológicos específicos para cada modalidade e para análise dos componentes corporais que interferem no alcance do alto rendimento ${ }^{13}$, sugerindo a importância de se chegar a um somatotipo adequado através de dieta e treinamento 5 .

Deste modo, este estudo teve como objetivo determinar e comparar o perfil antropométrico e somatotípico de jovens tenistas entre dois grupos maturacionais. Também foi objetivo deste estudo comparar as variáveis antropométricas entre o lado dominante e não-dominante, bem como comparar o perfil somatotípico calculado a partir de medidas obtidas no lado dominante e não-dominante em de jovens tenistas.

\section{Método}

\section{Sujeitos}

A amostra foi composta por 23 jogadores de tênis do sexo masculino residentes no estado de Santa Catarina, com idades variando entre 11-17 anos. Todos os participantes tinham no mínimo dois anos de experiência com o tênis competitivo. Os participantes e seus responsáveis foram informados de todos os procedimentos do experimento, assim como os riscos e benefícios, assinando um termo de consentimento livre e esclarecido, previamente aprovado pelo Comitê de Ética em Pesquisa envolvendo seres humanos da Universidade proponente.

Os participantes foram classificados em dois grupos a partir da maturação somática, a qual foi determinada indiretamente pela equação proposta por Mirwald et al. ${ }^{14}$. Esta equação identifica a distância do pico de velocidade de crescimento em estatura (PVE) de cada indivíduo em relação à idade cronológica em anos, sendo o critério de corte para dividir os grupos a distância $0 \mathrm{em}$ relação ao PVE (maturity offset). Os participantes do estudo que apresentaram valor inferior a 0 foram incluídos ao grupo denominado $\mathrm{PRE}_{\mathrm{PVE}}$ $(n=9)$, enquanto os sujeitos com valor maior ou igual à 0 foram classificados como PÓS $_{\mathrm{PVE}}$ $(\mathrm{n}=14)$. As características dos sujeitos de cada grupo são apresentadas na TABELA 1.

Em relação a técnica de golpes de backhand dos participantes, apenas um sujeito utiliza a técnica com uma das mãos.

\section{Medidas Antropométricas}

As avaliações foram realizadas seguindo técnicas padronizadas adotadas pelo International Society for the Advancement of Kinanthropometry $(\text { ISAK })^{15}$. Todas as medidas foram realizadas pelo mesmo avaliador, um antropometrista creditado pela ISAK. Todas as medidas segmentares foram realizadas em ambos os lados dos sujeitos.

As variáveis antropométricas incluíram massa corporal (MC), estatura (E), diâmetros (punho, úmero e fêmur), perímetros (braço contraído e panturrilha média) e dobras cutâneas (subescapular, supra ilíaca, tríceps e panturrilha). A estatura foi obtida com um estadiômetro com resolução de $0,1 \mathrm{~cm}$ (marca Sanny®) e a massa corporal foi mensurada utilizando-se uma balança eletrônica com resolução de $0,1 \mathrm{~kg}$ (marca Toledo®). As medidas de circunferência foram obtidas com uma fita métrica com escala de 0,1 mm (Cescorf®, Porto Alegre, Brasil). Para a mensuração de dobras cutâneas foi utilizado um adipômetro científico com resolução de 0,1 $\mathrm{mm}$ (Cescorf®, Porto Alegre, Brasil). Foram realizadas três medidas de cada dobra cutânea, sendo considerado o valor médio entre elas. O percentual de gordura corporal (\%GC) foi determinado a partir da equação proposta por Slaughter et al. ${ }^{16}$. O índice de massa corporal (IMC) também foi calculado.

O somatotipo foi determinado de acordo com as equações propostas por CARTER \& $\mathrm{HEATH}^{17}$. 
Para analises e comparações dos dados do somatotipo dos atletas recorreu-se ao cálculo da distância espacial entre os somatotipos (SAD - Somatotype Attitudinal Distance). Este valor representa a distância tridimensional entre dois somatopontos. Como critério para determinação da existência ou não de diferenças entre somatotipos utilizou-se $S A D \geq 1$. Cada unidade no valor do $\operatorname{SAD}(1,0)$ equivale à mudança de uma unidade na classificação de um componente $e^{17,18}$.

Tanto os componentes do somatotipo, quanto o percentual de gordura corporal foram calculados para ambos os lados, ou seja, estes índices foram calculados usando as medidas obtidas no lado direito e no lado esquerdo do participante, sendo caracterizados como dominante e não-dominante a partir do braço em que o jogador realiza o golpe de forehand.

\section{Análise Estatística}

Os dados estão apresentados como média e desvio padrão (DP). Para verificar a normalidade dos dados foi utilizado o teste de Shapiro-Wilk (intra-grupos e entre grupos). Para a comparação dos dados intra-grupo, utilizou-se o teste $t$ de student para amostra pareadas quando verificado a normalidade dos dados, no caso de a normalidade não ter sido verificada, utilizou-se e o teste não-paramétrico de Wilcoxon. Para a comparação dos dados entre grupos, utilizou-se o teste $t$ de student para amostras independentes. Quando a normalidade não foi verificada, foi realizada uma transformação logarítmica e nova verificação de normalidade, no caso de a normalidade não ser verificada novamente, utilizou-se o teste não-paramétrico de MannWhitney. Para todas as análises foi utilizado nível de significância de $5 \%(\mathrm{p}<0,05)$.

\section{Resultados}

A TABELA 1 apresenta as características físicas dos participantes separados a partir do ponto de corte do pico de velocidade da estatura (PVE) (maturity offset) e também o volume em horas de treino semanal. Não foram observadas diferenças significativas para o volume de treino semanal entre os grupos. Todos os sujeitos PÓS ${ }_{\mathrm{PVE}}$ alcançaram o pico de velocidade após os 14 anos de idade.

TABELA 1 -Características da amostra dividida pelo critério maturacional dos participantes.

\begin{tabular}{lcc}
\hline \multirow{2}{*}{ Características } & $\mathbf{P R E}_{\mathrm{PVE}}(\mathbf{n}=\mathbf{9})$ & PÓS $_{\mathrm{PVE}}(\mathbf{n}=\mathbf{1 4})$ \\
\cline { 2 - 3 } & Média $\pm \mathrm{DP}$ & Média $\pm \mathrm{DP}$ \\
\hline Idade (anos) & $12,80 \pm 0,97$ & $15,6 \pm 0,94^{*}$ \\
Distância do PVE & $-1,3 \pm 0,9$ & $1,44 \pm 0,93$ \\
Estatura (cm) & $160,20 \pm 9,64$ & $180,6 \pm 5,24^{*}$ \\
Massa Corporal (kg) & $48,2 \pm 7,29$ & $70,3 \pm 8,45^{*}$ \\
IMC (kg.m $\left.{ }^{-2}\right)$ & $18,69 \pm 1,22$ & $21,48 \pm 1,77^{*}$ \\
Volume de treino (h/sem) & $14,20 \pm 3,28$ & $20,6 \pm 12,08$ \\
\hline
\end{tabular}

${ }^{*} \mathrm{p}<0,001$ em comparação ao grupo $\mathrm{PRE}_{\mathrm{PVE}}$
A TABELA 2 apresenta as médias, desvio padrão e os resultados da análise estatística entre as dominâncias intra-grupos para os dados de diâmetros ósseos, perímetros musculares, dobras cutâneas e porcentagem de gordura. Além disso, apresenta as significâncias estatísticas quando as variáveis foram comparadas entre os grupos para os casos dominante vs dominante e não-dominante vs não-dominante.

$\mathrm{Na}$ TABELA 3 são apresentados os valores de cada componente do somatotipo. Também é apresentado o SAD entre o lado dominante e não-dominante nos grupos $\mathrm{PRE}_{\mathrm{PVE}}$ e PÓS ${ }_{\mathrm{PVE}}$. 
TABELA 2 -Valores Médios \pm desvio padrão das variáveis antropométricas obtidas nos lados dominantes e nãodominantes em ambos os grupos maturacionais.

\begin{tabular}{|c|c|c|c|c|c|c|}
\hline & \multicolumn{3}{|c|}{ PRE $_{\mathrm{PVE}}$} & \multicolumn{3}{|c|}{ PÓS $_{\mathrm{PVE}}$} \\
\hline & Dom & Não-dom & $\mathrm{p}$ & Dom & Não-dom & $\mathrm{p}$ \\
\hline \multicolumn{7}{|c|}{ Diâmetros Ósseos (mm) } \\
\hline Punho & $51,66 \pm 3,8$ & $49,98 \pm 2,6$ & 0,063 & $58,83 \pm 2,16^{* *}$ & $57,34 \pm 2,09^{* *}$ & 0,003 \\
\hline Úmero & $65,13 \pm 3,18$ & $63,22 \pm 3,68$ & 0,007 & $72,99 \pm 2,86 * *$ & $70,62 \pm 3,92$ ** & 0,001 \\
\hline Fêmur & $94,97 \pm 3,53$ & $95,36 \pm 3,34$ & 0,479 & $100,2 \pm 4,32^{* * *}$ & $99,18 \pm 5,25$ & 0,184 \\
\hline \multicolumn{7}{|l|}{ Perímetros (cm) } \\
\hline Braço contraído & $25,54 \pm 1,78$ & $24,88 \pm 1,81$ & 0,026 & $29,37 \pm 3,76^{*}$ & $28,49 \pm 3,6^{*}$ & 0,001 \\
\hline $\begin{array}{l}\text { Panturrilha } \\
\text { medial }\end{array}$ & $32,94 \pm 2,21$ & $33,47 \pm 2,35$ & 0,011 & $37,79 \pm 2,04^{* *}$ & $37,92 \pm 2,05^{* *}$ & 0,4 \\
\hline \multicolumn{7}{|c|}{ Dobras Cutâneas (mm) } \\
\hline Subescapular & $6,96 \pm 1,19$ & $6,88 \pm 1,1$ & 0,337 & $9,07 \pm 3,33$ & $8,7 \pm 2,91 *$ & 0,209 \\
\hline Supraíliaca & $9,11 \pm 2,72$ & $9,79 \pm 4,77$ & 0,734 & $11,05 \pm 4,47$ & $11,31 \pm 4,47$ & 0,492 \\
\hline Tríceps & $10,77 \pm 2,8$ & $11,24 \pm 3,42$ & 0,537 & $9,69 \pm 3,91$ & $10,76 \pm 3,47$ & 0,01 \\
\hline Panturrilha & $13,76 \pm 3,8$ & $13,60 \pm 3,99$ & 0,84 & $9,4 \pm 3,14^{* * * *}$ & $9,57 \pm 3,35^{* *}$ & 0,619 \\
\hline \multicolumn{7}{|l|}{$\% G C$} \\
\hline SLAUGHTER et al. ${ }^{16}$ & $15,00 \pm 3,08$ & $15,31 \pm 3,77$ & 0,630 & $14,01 \pm 5,53$ & $14,73 \pm 4,91$ & 0,068 \\
\hline
\end{tabular}

TABELA 3 -Valores médios \pm desvio padrão dos componentes do somatotipo obtidos nos lados dominantes e não-dominantes e SAD em ambos os grupos maturacionais.

${ }^{*} \mathrm{p}<0,05,{ }^{* *} \mathrm{p}<$ $0,001,{ }^{* * *} \mathrm{p}=0,006$, **** $\mathrm{p}=0,007$ quando comparado a mesma variável e lateralidade entre grupos.

${ }^{*} \mathrm{p}<0,05$ quando comparado a mesma variável e lateralidade entre grupos.

$$
\text { PRÉ }_{\text {PVE }} \quad \text { PÓS }_{\text {PVE }}
$$

\begin{tabular}{lcccccc} 
& Dominante & $\begin{array}{c}\text { Não-domi- } \\
\text { nante }\end{array}$ & $\mathrm{p}$ & Dominante & $\begin{array}{c}\text { Não-domi- } \\
\text { nante }\end{array}$ & $\mathrm{p}$ \\
\hline Endomorfia & $2,9 \pm 0,7$ & $3,0 \pm 0,9$ & 0,565 & $2,8 \pm 1,0 *$ & $2,9 \pm 0,9$ & 0,068 \\
Mesomorfia & $4,5 \pm 0,6$ & $4,3 \pm 0,5$ & 0,042 & $4,4 \pm 0,9$ & $4,0 \pm 0,9$ & $<0,001$ \\
Ectomorfia & $3,7 \pm 0,8$ & $3,7 \pm 0,8$ & - & $3,5 \pm 0,8$ & $3,5 \pm 0,4$ & - \\
\hline SAD & & 0,290 & & & 1,921 & \\
\hline
\end{tabular}

De acordo com a classificação somatotípica proposta por CARTER e HEATH ${ }^{17}$, ambos somatotipos do grupo PRÉ ${ }_{\mathrm{PVE}}$ (obtido pelas medidas do lado dominante e não-dominante) e a obtida pelo lado dominante no grupo $\mathrm{PÓS}_{\mathrm{PVE}}$ são categorizados como ectomesomorfos. Entretanto, quando foi calculado pelas medidas do lado não-dominante, $\mathrm{o}$ grupo $\mathrm{POS}_{\mathrm{PVE}}$ foi categorizado como mesomorfoectomorfo.

A somatocarta para os lados não-dominante e dominante de ambos os grupos é apresentada na FIGURA 1. 


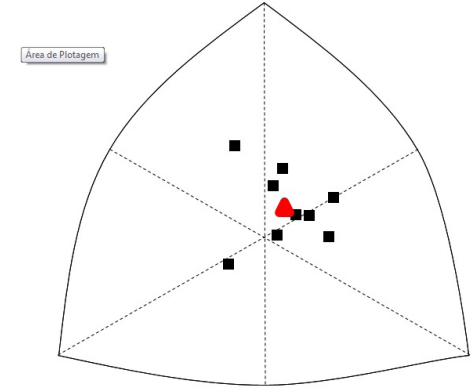

Somatocarta Não-Dominante PÓS

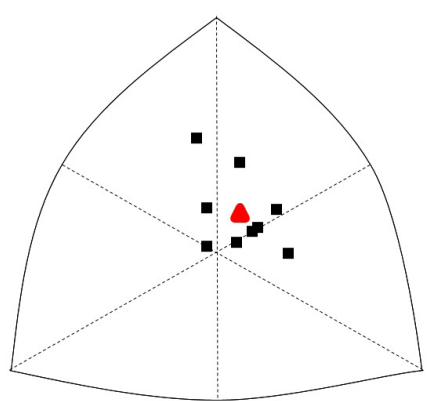

Somatocarta Dominante PÓS ${ }_{\mathrm{PVE}}$
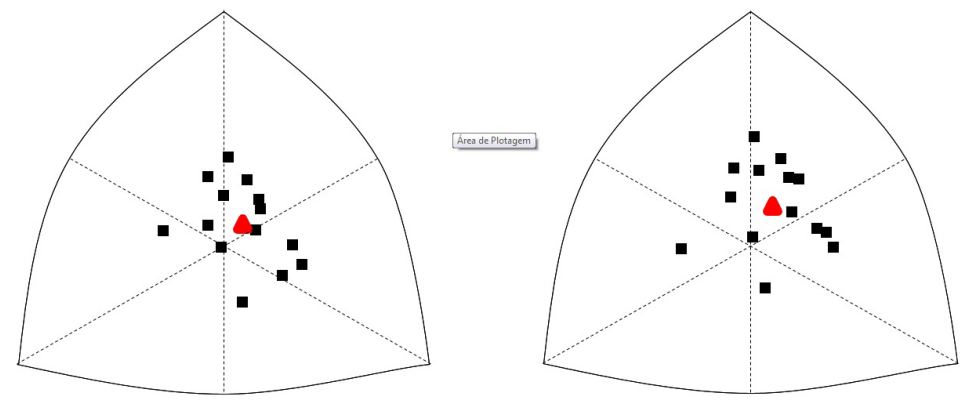

FIGURA 1 -Somatocarta obtida pelos lados dominante e não-dominante de cada grupo. Os somatopontos de cada sujeito estão representados pelo quadro. O somatoponto vermelho (triangulo) representa a média do grupo.

\section{Discussão}

Assimetrias de membros superiores em tenistas juvenis e profissionais têm sido reportado em diferentes estudos ${ }^{8,10,12,19-21}$. No presente estudo, em ambos os grupos foram observadas diferenças significantes de diâmetro ósseo de úmero e no perímetro do braço contraído, sendo os valores do lado dominante superiores. O grupo $\mathrm{PÓS}_{\mathrm{PVE}}$ também apresentou assimetria significativa de diâmetro ósseo de punho, enquanto que o grupo $\mathrm{PRE}_{\mathrm{PVE}}$ apresentou tendência estatística $(\mathrm{p}=0,06)$ para esta variável, o que nos permite inferir que a diferença de diâmetros entre punhos aumenta com o aumento da prática e com a maturação somática em jovens tenistas. De fato, o período de ocorrência do PVE é responsável por grandes alteraçôes em diversas variáveis fisiológicas e morfológicas, como o aumento nos níveis de força, potência, massa magra e gorda, densidade mineral óssea entre outras, sendo que essas variáveis diferem entre os $\operatorname{sexos}^{22-24}$.

Neste sentido, Silva et al. ${ }^{25}$ apresentaram evidências de que a idade entre 14 e 16 anos apresenta um aumento exponencial na mineralização óssea em meninos. Além disso, o treinamento físico durante a puberdade acarreta no incremento do conteúdo mineral ósseo, principalmente nos segmentos corpóreos nos quais ocorrem os esforços mecânicos ${ }^{26}$, como no caso do tênis, onde os golpes provocam tensôes mecânicas repetitivas no braço e antebraço dominante.

O pico de ganho de massa muscular, ocorre aproximadamente seis meses após o PVE, o qual está diretamente associado à elevação do hormônio testosterona ${ }^{22,27}$, explicando as diferenças encontradas nas medidas dos perímetros entre os grupos. Um achado interessante do presente estudo foi no perímetro da panturrilha medial para o grupo $\mathrm{PRE}_{\mathrm{PVE}}$, o qual apresentou assimetria significante sendo o lado não-dominante maior, entretanto, esta assimetria não foi observada no grupo $\mathrm{PÓS}_{\mathrm{PVE}}$.

Devido as poucas informaçóes sobre os tipos de treinamento aos quais os sujeitos são submetidos, especula-se que esta assimetria pode ser causada 
pela sobrecarga que o movimento do saque provoca sobre a perna não-dominante. Neste golpe, existe grandes amplitudes de flexão de joelho durante a fase inicial do movimento, valores variando entre $69^{\circ}$ e $85^{\circ}$ para o joelho não-dominante ${ }^{28,29}$. Na sequência do golpe, ocorre um aumento na força de reação dos pés contra o solo na componente vertical para que ocorra o momento de propulsão do saque, com magnitudes variando entre 1122 e 1753 $\mathrm{N}$ dependendo da técnica utilizada, em jogadores adultos $^{28,30}$. Durante a fase aérea, os tenistas chegam a sair do chão, em média, $4-7 \mathrm{~cm}$ e a aterrisagem ocorre novamente na perna não-dominante. Além disso, tenistas com a faixa etária media do grupo $\mathrm{PRE}_{\mathrm{PVE}}$ normalmente não realizam trabalhos de musculação, por esse motivo, acredita-se que a sobrecarga gerada pelo saque possa ser um fator de maior desenvolvimento muscular da panturrilha não-dominante. Porém, esta é uma variável que precisa ser melhor investigada.

Os tenistas avaliados neste estudo, apresentaram valores de IMC semelhantes aos valores reportados por outros estudos com tenistas jovens ${ }^{31,32}$, entretanto estes valores são menores com relação a jogadores profissionais, os quais apresentam um IMC de 23,5 kg. $\mathrm{m}^{-2} 5,33$.

Em determinadas populações de atletas, o IMC é um indicador válido de massa muscular ${ }^{34}$. GALEWatTs e NevilL ${ }^{33}$ realizam um mapeamento do IMC de jogadores participantes dos quatro torneios conhecidos como Grand Slam ou "Majors" entre os anos de 1982 e 2011 e, encontraram um aumento do IMC ao longo do tempo nos jogadores de elite. Além disso, maiores valores de IMC surgem como um importante fator associado com o sucesso. Os autores sugerem que o aumento da massa muscular pode conferir vantagem devido o perfil intermitente e de alta intensidade dos estímulos durante uma partida de tênis.

A estimativa do percentual de gordura da presente amostra apresentou índices semelhantes aos encontrados em tenistas juvenis de elite mundial ${ }^{31}$, porém estes valores ainda estão acima do recomendado por $\mathrm{KOVACS}^{4}$, o qual sugere que os tenistas deveriam se manter em um percentual de gordura inferior a $12 \%$. De acordo com JUZWIAK et al. ${ }^{35}$, um percentual de gordura reduzido pode ser vantajoso para aumentar a agilidade e velocidade de deslocamento em quadra, enquanto que um elevado percentual de gordura aumenta a possibilidade de desenvolver lesōes ${ }^{36}$.

O cálculo dos componentes do somatotipo foi orginalmente proposto por HEATH e CARTER ${ }^{37}$ a partir das medidas do lado direito do indivíduo. Entretanto, considerando que existem diferenças entre os lados dominante e não-dominante de tenistas, o cálculo a partir do lado direito pode conduzir a um possível viés de interpretação. Desta forma, o presente estudo comparou o somatotipo a partir de medidas obtidas em ambos os lados de jovens tenistas.

Para ambas as lateralidades o componente mesomórfico foi o maior para os dois grupos $\left(\mathrm{PRE}_{\mathrm{PVE}}\right.$ e PÓS $\left.{ }_{\mathrm{PVE}}\right)$. Esta característica também foi observada em outros estudos que analisaram tenistas jovens $s^{5,38,39}$ e adultos 5

O somatotipo médio do grupo PRÉ $\mathrm{PVE}_{\mathrm{P}}$ obtido pelo lado dominante e obtido pelo lado não-dominante e somatotipo obtido pelo lado dominante do grupo PÓ $_{\mathrm{PVE}}$ foram definidos como ectomesomorfo, caracterizando atletas com características de força, de grande estatura e lineares ${ }^{40}$. Este perfil também foi verificado nos estudos de SÁnCHEZ-MuÑoz, Sanz e Zabala ${ }^{20}$, Elliott et al. ${ }^{38}$, Dias et al. ${ }^{39}$, os quais avaliaram jovens tenistas. Entretanto, de acordo com as categorias propostas por CARTER ${ }^{18}$, o grupo de tenistas do estudo de SÁnCHEZ-MuÑoZ, SANZ E ZABALA $^{31}$ deveria ter sido classificado como mesomorfo balanceado, segundo os valores médios do somatotipo apresentado. Jogadores de elite avaliados por Martinez-Rodriguez, Collado E Vicente-Salar ${ }^{5}$ foram categorizados como endomesormorfos.

O somatotipo pode sofrer alterações durante todo processo maturacional e, também sofrer influências devido ao treinamento sistemático ${ }^{26,41}$. De fato, o lado dominante e não-dominante para o grupo PÓS ${ }_{\mathrm{PVE}}$ apresentaram somatotipos diferentes, ectomesomorfo e mesomorfoectomorfo, respectivamente. Esta diferença entre os somatotipos médios foi confirmada através do cálculo do SAD.

O componente mesomórfico (TABELA 3) foi o responsável direto pelos diferentes somatotipos, influenciado diretamente pelas diferenças significativas encontradas no diâmetro ósseo de úmero, perímetro de braço contraído e dobra cutânea de tríceps (TABELA 2). Apesar do grupo PRÉ também apresentar diferença significativa neste componente, esta não foi suficiente para provocar alterações nos somatotipos. Como mencionado por MaLINA et al. ${ }^{26}$, o somatotipo sofre poucas alterações durante o período maturacional, entretanto quando observada alterações, estas normalmente ocorrem 
por diferenças no componente de mesomorfia, principalmente em meninos.

Para nosso conhecimento, este foi o primeiro estudo a comparar o somatotipo calculado a partir de medidas obtidas do lado dominante e do lado não-dominante. CARTER E HEATH ${ }^{17}$ enfatizam que as medidas para o cálculo do somatotipo devem ser obtidas do lado direito do avaliado, entretanto, como verificado acima, em esportes com predominância unilateral deve-se ter cautela no cálculo do somatotipo a partir do lado direito, como é proposto originalmente, pois este pode estar fornecendo um resultado equivocado para um jogador canhoto. Uma sugestão, seria obter a medida de ambos os lados e realizar uma média para o cálculo do somatotipo.

Independentemente do nível maturacional, assimetrias de membros superiores foram observadas, sendo estas maiores no lado dominante em jovens tenistas. Além disso, este estudo fornece informações importantes acerca do perfil somatotípico de jovens tenistas e, com os cuidados que se deve ter para o cálculo do somatotipo em atletas praticantes de modalidades com predominância unilateral.

Por fim, o uso de medidas antropométricas, apesar de apresentar limitaçóes, pode ser considerado um instrumento valioso para treinadores, uma vez que apresenta baixo custo e fácil aplicabilidade. A partir de medidas antropométricas é possível estimar o estágio de maturação somática de jovens atletas, além de permitir o monitoramento e acompanhamento da composição corporal e de assimetrias morfológicas. Tais medidas permitem ao treinador ou preparador físico, determinar os tipos de treinamento de acordo com o estágio maturacional de seus jovens atletas.

\section{Abstract}

Anthropometrical and somatotype comparison of young tennis players from laterality

The aim of this study was to determine and compare the anthropometric profile and the somatotype of young tennis players classified into two maturational groups. It was also objective to compare the anthropometrical variables between the dominant and non-dominant sides, as well as to compare the somatotype profile obtained from measurements taken from both sides. The sample was composed by 23 male tennis players with age ranging from 11 to 17 years old. The participants were classified into two groups according to estimate of distance from the peak height velocity (PHV). That players which present values lower than 0 were allocated to group named PRÉ ${ }_{\mathrm{PVE}}(\mathrm{n}=9)$, whilst the players with values equal or higher to 0 were

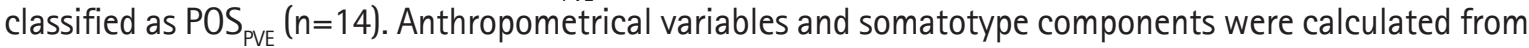
measurements taken at both sides of the body. Asymmetries (significant difference between sizes) were observed to the width of wrist bone and triceps skinfold for $\mathrm{POS}_{\mathrm{PVE}}$ and width of humerus bone and girth of contracted arm for both groups. The somatotype components derived from dominant side in the PRE ${ }_{\mathrm{PVE}}$ group were $2.9-4.5-3.7$, and non-dominant $3.0-4.3-3.7$, both were classified as ectomorphs. The POS $_{\text {PVE }}$ group was classified as ectomesomorph $(2.8-4.4-3.5)$ for dominant side, whilst for non-dominant was classified as mesomorph-ectomorph (2.9- 4.0 - 3.5). For sports with unilateral demand one should be caution on calculation of somatotype from the right side of body, as originally proposed. It could lead to misinterpretation of somatotype profile of athlete, especially for those with somatic maturation advanced.

KEYwords: Tennis; Anthropometric; Somatotype; Asymmetry; Body Composition.

\section{Referências}

1. Ziemann E, Sledziewska E, Grzywacz T, Gibson AL, Wierzba TH. Body composition and physical capacity of elite adolescent female tennis players. Georg Med News. 2011;(196-197):19-27.

2. Fernandez J, Mendez-Villanueva A, Pluim BM. Intensity of tennis match play. Br J Sports Med. 2006;40:387-91.

3. Mendez-Villanueva A, Fernandez-Fernandez J, Bishop D, Fernandez-Garcia B, Terrados N. Activity patterns, blood 
lactate concentrations and ratings of perceived exertion during a professional singles tennis tournament. Br J Sports Med. 2007;41:296-300.

4. Kovacs MS. Tennis physiology: training the competitive athlete. Sports Med. 2007;37:189-98.

5. Martinez-Rodriguez A, Collado ER, Vicente-Salar N. Estudio de la composición corporal de jugadores adultos de pádel y tenis. Nutr Hosp. 2015;31:1294-301.

6. Wilmore JH, Costill DL. Training for Sport and Activity: The Physiological Basis of the Conditioning Process. 3rd ed. Human Kinetics; 1993. p. 420.

7. Baxter-Jones AD, Helms P, Maffulli N, Baines-Preece JC, Preece M. Growth and development of male gymnasts, swimmers, soccer and tennis players: a longitudinal study. Ann Hum Biol. 1995;22:381-94.

8. Rogowski I, Ducher G, Brosseau O, Hautier C. Asymmetry in volume between dominant and nondominant upper limbs in young tennis players. Pediatr Exerc Sci. 2008;20:263-72.

9. Saccol MF, Gracitelli GC, da Silva RT, Laurino CF de S, Fleury AM, Andrade M dos S, et al. Shoulder functional ratio in elite junior tennis players. Phys Ther Spor. 2010;11:8-11.

10. Sanchis-Moysi J, Dorado C, Olmedillas H, Serrano-Sanchez JA, Calbet JAL. Bone and lean mass inter-arm asymmetries in young male tennis players depend on training frequency. Eur J Appl Physiol. 2010;110:83-90.

11. Ducher G, Jaffré C, Arlettaz A, Benhamou C-L, Courteix D. Effects of long-term tennis playing on the muscle-bone relationship in the dominant and nondominant forearms. Can J Appl Physiol. 2005;30:3-17.

12. Sanchís-Moysi J, Idoate F, Olmedillas H, Guadalupe-Grau A, Alayón S, Carreras A, et al. The upper extremity of the professional tennis player: muscle volumes, fiber-type distribution and muscle strength. Scand J Med Sci Sports. 2010;20:524-34.

13. Carter JEL, Ackland TR, Kerr DA, Stapff AB. Somatotype and size of elite female basketball players. J Sports Sci. 2005;23:1057-63.

14. Mirwald RL, Baxter-Jones ADG, Bailey DA, Beunen GP. An assessment of maturity from anthropometric measurements. Med Sci Sports Exerc. 2002;34:689-94.

15. Stewart TOA, Marfell-Jones LCM, ISAK. International Standards for Anthropometric Assessment. Glasgow: International Society for the Advancement of Kinanthropometry; 2006.

16. Slaughter MH, Lohman TG, Boileau RA, Horswill CA, Stillman RJ, Van Loan MD, et al. Skinfold equations for estimation of body fatness in children and youth. Hum Biol . 1988;60:709-23.

17. Carter JEL, Heath BH. Somatotyping - Development and Applications. 1st ed. New York: Cambridge; 1990.

18. Carter JEL. Part 1: The Heath-Carter Anthropometric Somatotype. Somatotype Instruction Manual. San Diego, CA: J.E.L. Carter; 2002.

19. Ducher G, Courteix D, Même S, Magni C, Viala JF, Benhamou CL. Bone geometry in response to long-term tennis playing and its relationship with muscle volume: a quantitative magnetic resonance imaging study in tennis players. Bone. 2005;37:457-66.

20. Sanchis-Moysi J, Dorado C, Idoate F, González-Henríquez JJ, Serrano-Sanchez JA, Calbet JAL. The asymmetry of pectoralis muscles is greater in male prepubertal than in professional tennis players. Eur J Sport Sci. 2016;16(7):780-6.

21. Sanchis-Moysi J, Idoate F, Dorado C, Alayón S, Calbet JAL. Large asymmetric hypertrophy of rectus abdominis muscle in professional tennis players. PLoS One. 2010;5:e15858.

22. Beunen G, Thomis M. Muscular Strength Development in Children and Adolescents. Pediatr Exerc Sci. 2000;12:174-97.

23. Van Praagh E. Development of Anaerobic Function during Childhood and Adolescence. Pediatr Exerc Sci. 2000;12:150-73.

24. Armstrong N, Welsman JR. Development of Aerobic Fitness during Childhood and Adolescence. Pediatr Exerc Sci. 2000;12:128-49.

25. Silva CC, Goldberg TBL, Teixeira AS, Dalmas JC. Mineralização óssea em adolescentes do sexo masculino : anos críticos para a aquisição da massa óssea. J Pediatr (Rio J). 2004;80:461-7.

26. Malina R, Bouchard C, Bar-Or O. Growth, Maturation \& Physical Activity. 2nd ed. Champaign: Human Kinetics; 2004. p. 728.

27. Rogol AD, Clark PA, Roemmich JN. Growth and pubertal development in children and adolescents: effects of diet and physical activity. Am J Clin Nutr. 2000;72:521S-8S.

28. Braga Neto L. Estudo de Características Dinâmicas e Eletromiográficas do Saque no Tênis - Comparação Entre Duas Técnicas. São Paulo: Universidade de São Paulo; 2003.

29. Reid M, Elliott B, Alderson J. Lower-limb coordination and shoulder joint mechanics in the tennis serve. Med Sci Sports 
Exerc [Internet]. 2008 [cited 2013 Apr 6];40:308-15. Available from: http://www.ncbi.nlm.nih.gov/pubmed/18202570.

30. Gheluwe B Van, Hebbelinck M. Muscle Actions and Ground Reaction Forces in Tennis. Int J Sport Biomech. 1986;2:88-100.

31. Sánchez-Muñoz C, Sanz D, Zabala M. Anthropometric characteristics, body composition and somatotype of elite junior tennis players. Br J Sports Med. 2007;41:793-9.

32. Cócaro ES, Priore SE, Costa RF da, Fisberg M. Consumo alimentar e perfil antropométrico de adolescentes tenistas. Rev da Soc Bras Aliment e Nutr. 2012;37:293-308.

33. Gale-Watts AS, Nevill AM. From endurance to power athletes: The changing shape of successful male professional tennis players. Eur J Sport Sci. 2016;1391:1-7.

34. Nevill AM, Winter EM, Ingham S, Watts A, Metsios GS, Stewart AD. Adjusting athletes' body mass index to better reflect adiposity in epidemiological research. J Sports Sci. 2010;28:1009-16.

35. Juzwiak CR, Amancio OMS, Vitalle MSS, Pinheiro MM, Szejnfeld VL. Body composition and nutritional profile of male adolescent tennis players. J Sports Sci. 2008;26:1209-17.

36. Tyler TF, McHugh MP, Mirabella MR, Mullaney MJ, Nicholas SJ. Risk factors for noncontact ankle sprains in high school football players: the role of previous ankle sprains and body mass index. Am J Sports Med. 2006;34:471-5.

37. Heath BH, Carter JEL. A modified somatotype method. Am J Phys Anthropol. 1967;27:57-74.

38. Elliott BC, Ackland T, Blanksby B, Bloomfield J. A prospective study of physiological indicators of junior tennis performance. Aust J Sci Med Sport. 1990;22:87-92.

39. Dias C, Junior DA, Candido Z, Netto DO, Cesar J. Perfil morfofisiológico e somatotipológico de jovens atletas da modalidade tênis de campo. Rev UNIANDRADE. 2013;14:229-40.

40. Cabral BG de AT, Cabral S de AT, de Miranda HF, Dantas PMS, Reis VM. Efeito discriminante da morfologia e alcance de ataque no nível de desempenho em voleibolistas. Rev Bras Cineantropometria e Desempenho Hum. 2011;13:223-9.

41. Mortatti A, Arruda M. Análise do efeito do treinamento e da maturação sexual sobre o somatotipo de jovens futebolistas. Rev Bras Cineantropometria e Desempenho Hum. 2007;9:84-91.

ENDEREÇO DE CORRESPONDÊNCIA: Geovane Krüger

Rua Roberto Sampaio Gonzaga, Bloco V - Trindade

Florianópolis - SC - BRASIL

CEP: 88040-900

E-mail: geovanek@gmail.com; gktenis@gmail.com
Submetido: 08/09/2016

Revisado: 19/10/2017

Aceito: 19/06/2018 